\title{
ON A CLASS OF LIE ALGEBRAS
}

ROBERT H. OEHMKE

In 1958, Block constructed a new class $\mathfrak{B}$ of simple Lie algebras, (S) $(G, \delta, f)[1]$. Here $\&$ is the direct sum of a finite number of finite elementary $p$-groups, $G_{0}, \cdots, G_{n}, p>2$ and $\delta=\delta_{1}+\cdots+\delta_{n}$ where $\delta_{i}$ is a nonzero element of $G_{i}$. Let $F$ be a field of characteristic $p$. Then $f$ is a nondegenerate skew-symmetric biadditive form defined on each $G_{i}$ by $f_{i}(\alpha, \beta)=g_{i}(\alpha) h_{i}(\beta)-g_{i}(\beta) h_{i}(\alpha)$ for $\alpha, \beta$ in $G_{i}$ and where $g_{i}$ and $h_{i}$ are additive functions on $G_{i}$ to $F$ with $g_{i}\left(\delta_{i}\right)=0$. To each $\alpha \neq 0,-\delta$ of $G$ the formal symbol $v(\alpha)$ is assigned. Then $\mathfrak{R}(G, \delta, f)$ is the vector space over $F$ with the $v(\alpha)$ 's as a basis. The multiplication in $\&(G, \delta, f)$ is defined by

$$
v(\alpha) v(\beta)=\sum_{i=0}^{n} f_{i}\left(\alpha_{i}, \beta_{i}\right) v\left(\alpha+\beta-\delta_{i}\right)
$$

where $\alpha_{i}$ and $\beta_{i}$ are the components of $\alpha$ and $\beta$ in $G_{i}$ and where $\delta_{0}$ and $v(0)$ denote 0 .

Schafer [5] showed that each of these Lie algebras can be realized as the derived algebra of the algebra of inner derivations of a simple, nodal, Lie-admissible noncommutative Jordan algebra $A$. If $\mathfrak{R}$ is the class of all such Lie algebras that can be realized in this manner then Schafer's result is that $\mathfrak{\supseteq} \mathfrak{B}$. The main result of this paper is to show that $\mathbb{R}$ contains $\mathfrak{B}$ properly.

1. In this and subsequent sections $A$ will denote a finite dimensional, simple, nodal, Lie-admissible, noncommutative Jordan algebra over a field $F$ of characteristic $p>2$. Define $A^{+}$to be the algebra that is the same vector space as $A$ but has a product $x \circ y$ defined in terms of the product $x y$ of $A$ by

$$
x \circ y=\frac{1}{2}(x y+y x) \text {. }
$$

Define $A^{-}$to be the algebra that is the same vector space as $A$ but has a product $[x, y]$ defined in terms of the product $x y$ by

$$
[x, y]=x y-y x \text {. }
$$

Kokoris [3] has shown that $A^{+}$is the commutative, associative algebra $F\left[x_{1}, \cdots, x_{n}\right]$ of polynomials in $x_{1}, \cdots, x_{n}$ over $F$ with the restriction that $x_{i}^{p}=0$. Hence $A^{+}=F 1+N$ where $N$ is the set of nilpotent elements of $A^{+}$.

Received by the editors June 29, 1964. 
The multiplication in $A$ can be given by

$$
f g=f \circ g+\frac{1}{2} \sum \frac{\partial f}{\partial x_{i}} \circ \frac{\partial g}{\partial x_{j}} \circ c_{i j}
$$

where $\partial / \partial x_{i}$ are the ordinary partial differential operators and $c_{i j}$ $=x_{i} x_{j}-x_{j} x_{i}$. We shall confine our attention to the case $n=2$. In [5] it was shown that a pair of generators $x$ and $y$ could be chosen for $A^{+}$such that

$$
y x-x y=y D(x)=1+\alpha x^{p-1} \circ y^{p-1}
$$

for some $\alpha$ in $F$. By the multiplication given in (1) we see that $\alpha$ completely determines the algebra $A$.

Theorem 1. If $A_{1}$ and $A_{2}$ are two algebras such that $A_{1}^{+}$and $A_{2}^{+}$have two generators and if they are defined by

$$
v D_{1}(x)=1+\alpha_{1} x^{p-1} \cdot y^{p-1}
$$

and

$$
v D_{2}(u)=1+\alpha_{2} u^{p-1} \cdot v^{p-1}
$$

respectively then $A_{1}$ and $A_{2}$ are isomorphic if and only if $\alpha_{1}=\alpha_{2}$.

Proof. The sufficiency of the condition $\alpha_{1}=\alpha_{2}$ is of course trivial. Therefore we shall assume $A_{1}$ and $A_{2}$ are isomorphic. In fact we can assume $A=A_{1}=A_{2}$ and $(x, y)$ and $(u, v)$ are two pairs of generators for $A^{+}$. Then both $u$ and $v$ can be expressed as polynomials in $x$ and $y$. Jacobson [2] has shown that any representatives in $A^{+}$of the elements of any basis of the 2 dimensional space $N / N \circ N$ will serve as a pair of generators. Hence if $x$ and $y$ are generators of $A^{+}$then so also are $x_{1}=x+f(y)$ and $y_{1}=y$ where $f(y)$ is of degree at least 1 in $y$. For if the cosets with representatives $x$ and $y$ form a basis for the space $N / N \circ N$ then so also do the cosets with representatives $x+\alpha y$ and $y$ for any $\alpha$ in $F$. Clearly, this pair of generators will also satisfy (3) for the same $\alpha_{1}$ since $y^{i} D_{1}(y)=0$ and $x_{1}^{p-1} \circ y_{1}^{p=1}=x^{p-1} \circ y^{p-1}$. In the same manner, we can replace $x_{1}$ and $y_{1}$ by $x_{2}=x_{1}$ and $y_{2}=y_{1}$ $+g\left(x_{1}\right)$ and still retain (3).

If $u=\delta_{1} x+\delta_{2} y+h(x, y)$ and $v=\delta_{3} x+\delta_{4} y+q(x, y)$ where $h(x, y)$ and $q(x, y)$ are of degree at least 2 in $x$ and $y$ then, since $v D_{2}(u)=1$ $+\alpha_{2} u^{p-1} \circ v^{p-1}$, we have $\delta_{1} \delta_{4}-\delta_{2} \delta_{3}=1$. Therefore either $\delta_{1} \delta_{4} \neq 0$ or $\delta_{2} \delta_{3} \neq 0$. Without loss of generality we can assume that the coefficient of $x$ in $u$ and the coefficient of $y$ in $v$ is not zero. For if $\delta_{1} \delta_{4}=0$ we can replace $u$ and $v$ by $u_{1}=-v$ and $v_{1}=u$ and still retain (2). Now by a suitable choice of the functions $f(y)$ and $g\left(x_{1}\right)$ above we can assume 
there is a pair of generators $x_{2}, y_{2}$ such that

$$
y_{2} D_{1}\left(x_{2}\right)=1+\alpha_{1} x_{2}^{p-1} \circ y_{2}^{p-1}
$$

and

$$
\begin{aligned}
& u=\delta_{1} x_{2}+h^{\prime}\left(x_{2}, y_{2}\right) \circ x_{2}, \\
& v=\delta_{1}^{-1} y_{2}+q^{\prime}\left(x_{2}, y_{2}\right) \circ y_{2},
\end{aligned}
$$

where $h^{\prime}$ and $q^{\prime}$ are of degree at least 1 in $x_{2}$ and $y_{2}$.

We shall assume $x$ and $y$ are such a pair of generators. We have $u^{p-1} \circ v^{p-1}=\delta_{1}^{p-1} \delta_{1}^{-p+1} x^{p-1} \circ y^{p-1}=x^{p-1} \circ y^{p-1}$. Write

$$
\begin{aligned}
& u=\sum_{i, j=0}^{p-1} \alpha_{i j} x^{i} \circ y^{j}, \\
& v=\sum_{i, j=0}^{p-1} \beta_{i j} x^{i} \circ y^{j}
\end{aligned}
$$

and note that $\alpha_{0 j}=\beta_{i 0}=0$. The coefficient of $x^{p-1} \circ y^{p-1}$ in the expression

$$
\begin{aligned}
\vartheta D_{2}(u) & =\sum_{i, j, s, t=0}^{p-1}(i t-j s) \alpha_{i j} \beta_{s t} x^{i+o-1} \circ y^{j+t-1} \circ\left(1+\alpha_{1} x^{p-1} \circ y^{p-1}\right) \\
& =1+\alpha_{2} u^{p-1} \circ v^{p-1}=1+\alpha_{2} x^{p-1} \circ y^{p-1}
\end{aligned}
$$

will occur on the left only if either (a) $i+s-1=j+t-1=0$ or (b) $i+s$ $-1=j+t-1=p-1$. If $(\mathrm{a})$, then the coefficient is $\left(\alpha_{10} \beta_{01}-\alpha_{01} \beta_{10}\right) \alpha_{1}$. But $\alpha_{10}=\delta_{1}=\beta_{01}^{-1}$ and $\beta_{10}=\alpha_{01}=0$. Therefore such a term will have a coefficient $\alpha_{1}$. If (b), then $i \equiv-s$ and $j \equiv-t$ modulo $p$ and (it $-j s$ ) $\equiv 0$ modulo $p$. Hence we must have $\alpha_{1}=\alpha_{2}$ and the proof is complete.

2. Schafer [5] has shown that the algebra $A$ associated with the algebra $\mathbb{S}(G, \delta, f)$ (if $A^{+}$has only two generators) has generators $x$ and $y$ such that either

$$
y D(x)=\beta(1+x) \circ(1+y)
$$

or

$$
y D(x)=\beta
$$

for some nonzero $\beta$ in $F$. In the latter case by replacing $x$ by $\beta^{-1} x$ we see that $A$ is an algebra satisfying (2) with $\alpha=0$. In the former case, Schafer has shown [5, p. 322] that $A$ is an algebra that satisfies (2) with $\alpha=-\beta^{p-1}$. We let $\mathfrak{D}(A)$ be the set of inner derivations of $A$ and $\mathfrak{D}^{\prime}(A)$ the derived algebra of the algebra of inner derivations of 
$A$. Clearly if $A_{1}$ and $A_{2}$ are isomorphic so will $\mathfrak{D}^{\prime}\left(A_{1}\right)$ and $\mathfrak{D}^{\prime}\left(A_{2}\right)$ be isomorphic. However, the following theorem shows that the converse does not hold for arbitrary fields $F$.

Theorem 2. If $A_{1}$ and $A_{2}$ are two algebras defined by the field elements $\alpha_{1}$ and $\alpha_{2}$ respectively then $\mathfrak{D}^{\prime}\left(A_{1}\right)$ and $\mathfrak{D}^{\prime}\left(A_{2}\right)$ are isomorphic if and only if there is a nonzero $\delta$ in $F$ such that $\alpha_{1}=\delta^{p-1} \alpha_{2}$.

Proof. Assume $x$ and $y$ are generators of $A_{1}^{+}$such that $y D_{1}(x)$ $=1+\alpha_{1} x^{p-1} \circ y^{p-1}$. We can assume that $\alpha_{1} \neq 0$. For if $\alpha_{1}=0$ then the dimension of $\mathfrak{D}^{\prime}\left(A_{1}\right)$, and hence $\mathfrak{D}^{\prime}\left(A_{2}\right)$, is $p^{2}-2$ [5, Theorem 6]. Therefore $\alpha_{2}=0$ and $A_{1}$ and $A_{2}$ are isomorphic. Now if $\alpha_{1} \neq 0$ then $\mathfrak{D}^{\prime}\left(A_{1}\right) \cong \mathfrak{D}\left(A_{1}\right) \cong A_{1}^{-} / F 1[5$, p. 320].

Let $\sigma$ be an isomorphism from $A_{2}^{-} / F 1$ onto $A_{2}^{-} / F 1$. Since each element of $A_{i}^{-} / F 1$ is a coset of the ideal $F 1$ of $A_{i}^{-}$and contains a unique element of $N_{i}$ we can consider $\sigma$ as a mapping of $N_{1}$ onto $N_{2}$. We let $\sigma\left(x^{i} \circ y^{i}\right)=z_{i j}$. (When convenient we shall use the symbol " $\equiv$ " to indicate the congruence relation induced in $A_{i}^{-}$by the ideal $F 1$ ).

LEMmA 1. If $s, t \in A_{i}^{-}$and $s D_{i}(t) \equiv 0$ then $s D_{i}(t)=0$.

Proof. If $s D_{i}(t) \equiv 0$ then there is a $\delta \in F$ such that $s D_{i}(t)=\delta$. Assume $\delta \neq 0$. Then $s$ and $t$ must be a pair of generators of $A_{i}^{+}$. But this implies that ( 7$)$ is satisfied contradicting our assumption above.

We return to the proof of the theorem. Since the elements $x^{i} \circ y^{j}$, $0 \leqq i, j \leqq p-1$ with not both $i$ and $j$ equal to zero, form a basis for the vector space $N_{1}$, the elements $z_{i j}$ form a basis for the vector space $N_{2}$. Hence there must be a pair, say $u=z_{s t}$ and $v=z_{m n}$, that are generators of $A_{2}^{+}$. Assume that both $\max (s, t)>1$ and $\max (m, n)>1$. Then

$$
\begin{aligned}
& \left(x^{s} \circ y^{t}\right) D_{1}\left(x^{p-1} \circ y^{p-1}\right)=(s-t) x^{p+o-2} \circ y^{p+t-2}=0, \\
& \left(x^{m} \circ y^{n}\right) D_{1}\left(x^{p-1} \circ y^{p-1}\right)=(m-n) x^{p+m-2} \circ y^{p+n-2}=0 .
\end{aligned}
$$

But since $\sigma$ is an isomorphism on $A_{1}^{-} / F 1$ we must have

$$
u D_{2}\left(z_{p-1, p-1}\right) \equiv v D_{2}\left(z_{p-1, p-1}\right) \equiv 0 .
$$

By Lemma 1 we have

$$
u D_{2}\left(z_{p-1, p-1}\right)=v D_{2}\left(z_{p-1, p-1}\right)=0 .
$$

By (1) we see that $w D_{2}\left(z_{p-1, p-1}\right)=0$ for all $w \in A_{2}$. It follows that $z_{p-1, p-1}=0$ [5, Lemma 2]. This is of course a contradiction of the definition of $z_{p-1, p-1}$. Hence we must have either $s, t \leqq 1$ or $m, n \leqq 1$. Say $s, t \leqq 1$. Again by (8) we can not have $s=t$. So assume $s=1$ and $t=0$ to get $\sigma(x)=u$. 
Now using $z_{p-1,0}$ in the same way we used $z_{p-1, p-1}$ we see that we must have $m \leqq 1$. By direct computation we see that there are two types of terms that annihilate $x^{m} \circ y^{n}$ in $A_{1}^{-} / F 1$. These are either of the form $w=x^{i m} \circ y^{i n}$ or $x^{i} \circ y^{p+1-i}$ for $j \leqq n$. For

$$
\left(x^{m} \cdot y^{n}\right) D_{1}\left(x^{i} \cdot y^{k}\right)=(i n-m k) x^{m+i-1} \cdot y^{n+k-1}=0
$$

if and only if either $i n-m k=0$ or $n+k-1 \geqq p$. No matter if $m=0$ or 1 , the first possibility holds precisely for these terms of the form $x^{i m} \circ y^{i n}$ for any nonnegative integer $i$. Clearly, the second possibility holds precisely for those terms of the form $x^{i} \circ y^{p+1-i}$ where $j \leqq n$ and $i$ is arbitrary. Hence if $n \geqq 2$ the subspace generated in $A_{1}^{-}$by such $w$ 's is of dimension $p(n-1)+r$ where $r$ is the number of independent terms of the form $x^{i m} \circ y^{i n}$. Therefore the dimension of the subspace of elements in $A_{2}^{-} / F 1$ that annihilate $v$ must be $p(n-1)+r$. However, if $z=\sum \beta_{i j} u^{i} \circ v^{i}$ and $z D_{2}(v) \equiv 0$ then we must have $\sum i \beta_{i j} u^{i-1}$ $\circ v^{j} \circ u D_{2}(v)=0$. Since $A_{2}$ is simple $u D_{2}(v)$ must be nonsingular [4]. Therefore $\sum i \beta_{i j} u^{i-1} \circ v^{j}=0$ and $z$ is a polynomial in $v$. But the subspace generated in $A_{2}-F 1$ by such $z$ 's is $p-1$. Therefore $n<2$ and $\sigma^{-1}(v)$ is either $x \circ y$ or $y$. Assume $\sigma^{-1}(v)=x \circ y$. Then $(x \circ y) D_{1}(x)=x$ so we must have $v D_{2}(u) \equiv u$ and $v D_{2}(u)=\delta+u$ for some $\delta \in F$. Since $v D_{2}(u)$ must be nonsingular we have $\delta \neq 0$ and $\left[v \circ(\delta+u)^{-1}\right] D_{2}(u)$ $=1$. But as argued above we see that such an assumption gives rise to a contradiction. Hence $\sigma^{-1}(v)=y$.

Recall that above we showed that the only polynomials that annihilate $v$ were the polynomials in $v$. Hence it follows that $\sigma\left(y^{i}\right)=f_{i}(v)$ is a polynomial in $v$. Analogously, $\sigma\left(x^{i}\right)=g_{i}(u)$ is a polynomial in $u$. Conversely, by arguing on the dimension of the subspace generated by the powers of $y$ we see that $\sigma^{-1}\left(v^{i}\right)=f_{i}^{\prime}(y)$, a polynomial in $y$, and $\sigma^{-1}\left(u^{i}\right)=g_{i}^{\prime}(x)$, a polynomial in $x$. We must have for $i>1$ that

$$
\sigma\left(x^{i-1}\right)=\sigma\left(x^{i} D_{1}(y)\right) \equiv g_{i}(u) D_{2}(v)=\frac{\partial g_{i}(u)}{\partial u} \circ u D_{2}(v)
$$

is a polynomial in $u$. Therefore, since $u^{2}$ is a linear combination of the $g_{i}$ 's we must have $u \circ u D_{2}(v)$ a polynomial in $u$ also. But then $u D_{2}(v)$ $=h_{1}(u)+u^{p-1} \circ h_{2}(v)$. Since a similar restriction holds for $v D_{2}(u)$ we must have

$$
v D_{2}(u)=\delta_{1}+\delta_{2} u^{p-1} \circ v^{p-1} \text {. }
$$

We shall now show by induction on the sum $i+j$ that

$$
\sigma\left(x^{i} \circ y^{j}\right)=\delta_{1}^{-i-j+1} u^{i} \circ v^{j} .
$$


Clearly, (9) holds if $i+j=1$. Also, if $i+j>1$ and $j>0$ then

$$
\begin{aligned}
\sigma\left(j x^{i} \circ y^{j-1}\right) & =\sigma\left(\left[x^{i} \circ y^{j}\right] D_{1}(x)\right) \equiv \sigma\left(x^{i} \circ y^{j}\right) D_{2}(u) \\
& =\partial \sigma\left(x^{i} \circ y^{j}\right) / \partial v \circ v D_{2}(u) \equiv \delta_{1}^{-i-j+2} u^{i} \circ v^{j-1} .
\end{aligned}
$$

Therefore $\delta_{1} \partial \sigma\left(x^{i} \circ y^{j}\right) / \partial v+\mu \delta_{2} u^{p-1} \circ v^{p-1} \equiv j \delta_{1}^{-i-j+1} u^{i} \circ v^{i-1}$ where $\mu$ is the coefficient of $v$ in $\sigma\left(x^{i} \circ y^{j}\right)$. Since $\mu \delta_{2} u^{p-1} \circ v^{p-1}$ is the only term of degree $p-1$ in $v$ we have $\mu=0$. It follows that $\sigma\left(x^{i} \circ y^{j}\right)=\delta_{1}^{-i-j+1} u^{i}$ $\circ v^{i} \circ h(u)$. If $i=0$ then $\sigma\left(y^{j}\right)$ is a polynomial in $v$. Hence $h(u)$ is a constant $\beta$. If the constant $\beta$ is nonzero then $z_{i j}$ and $u$ are generators of $A_{2}^{+}$. But this implies, repeating the argument presented in this proof that $\sigma^{-1}\left(z_{i j}\right)=\epsilon y$ for some $\epsilon$ in $F$. Hence $z_{i j}=v, \beta=0$ and the induction holds if $i=0$. If $i \neq 0$ we can repeat the above argument using $\sigma\left(x^{i} \circ y^{j}\right) D_{2}(y)$ to get $\sigma\left(x^{i} \circ y^{j}\right)=\delta_{1}^{-i-j+1} u^{i} \circ v^{i}$. Therefore (9) holds for all $i$ and $j$. However, $\alpha_{1} \sigma\left(x^{p-1} \circ y^{p-1}\right) \equiv v D_{2}(y) \equiv \delta_{2} u^{p-1} \circ v^{p-1}$ since $y D_{1}(x) \equiv \alpha_{1} x^{p-1} \circ y^{p-1}$. But then $\alpha_{1} \delta_{1}^{-2 \rho+3}=\delta_{2}$. Now replace the generators $u$ and $v$ in $A_{2}^{+}$by $u^{\prime}=\delta_{1}^{-1} u$ and $v^{\prime}=v$ to get

$$
\begin{aligned}
v^{\prime} D_{2}\left(u^{\prime}\right) & =\delta_{1}^{-1}\left(v D_{2}(u)\right)=1+\delta_{1}^{-1} \delta_{2} u^{p-1} \circ v^{p-1} \\
& =1+\alpha_{1} \delta_{1}^{-2 p+2} u^{p-1} \circ v^{p-1}=1+\alpha_{1} \delta_{1}^{-p+1}\left(\delta_{1}^{-1} u\right)^{p-1} \circ v^{p-1} \\
& =1+\alpha_{1} \delta_{1}^{-p+1} u^{p-1} \circ v^{p-1} .
\end{aligned}
$$

Hence the necessity of the condition for an isomorphism holds.

Conversely, let $x$ and $y$ be generators of $A_{1}^{+}$and $u$ and $v$ be generators of $A_{2}^{+}$such that

$$
\begin{aligned}
& y D_{1}(x)=1+\alpha_{1} x^{p-1} \circ y^{p-1} \\
& v D_{2}(u)=1+\alpha_{1} \delta_{1}^{-p+1} u^{p-1} \circ v^{p-1}
\end{aligned}
$$

for some nonzero $\delta_{1} \in F$. Define the linear mapping $\sigma$ from $A_{1}$ to $A_{2}$ on the basal elements $x^{i} \circ y^{j}$ by

$$
\sigma\left(x^{i} \circ y^{j}\right)=\delta_{1}^{-j+1} u^{i} \circ v^{j} \text {. }
$$

A straightforward computation shows that $\sigma$ is an isomorphism of $A_{1}^{-} / F 1$ onto $A_{2}^{-} / F 1$. As noted above each algebra of $\mathfrak{B}$ can be obtained from an algebra $A$ satisfying (2) with either $\alpha=0$ or $\alpha=-\beta^{p-1}$ for some $\beta \in F$. It was shown [4, Theorem 3] that those algebras of $\mathfrak{B}$ with the corresponding $\alpha=0$ or $-\beta^{p-1}$ are of dimension $p^{2}-2$ and $p^{2}-1$ respectively. Therefore there are at least two nonisomorphic algebras in $\mathfrak{B}$. However from Theorem 2 we see that all of the algebras of $\mathfrak{B}$ obtained from an algebra $A$ with $\alpha=-\beta^{p-1}$ are isomorphic. Hence

COROLlaRY. There are two nonisomorphic types of algebras in $\mathfrak{B}$ cor- 
responding to an $A$ such that $A^{+}$has two generators; one of dimension $p^{2}-1$ and one of dimension $p^{2}-2$.

To construct an algebra in $\mathbb{R}$ but not in $\mathfrak{B}$ we need only choose a field $F$ containing an element $\alpha$ such that $x^{p-1}+\alpha$ is irreducible over F.

CoRollary. The class $\mathfrak{B}$ is properly contained in in the class $\mathbb{R}$.

\section{REFERENCES}

1. Richard Block, New simple Lie algebras of prime characteristic, Trans. Amer. Math. Soc. 89 (1958), 421-449.

2. Nathan Jacobson, Classes of restricted Lie algebras of characteristic p. II, Duke Math. J. 10 (1943), 107-121.

3. L. A. Kokoris, Nodal noncommutative Jordan algebras, Canad. J. Math. 12 (1960), 488-492.

4. Robert H. Oehmke, Nodal noncommutative Jordan algebras, Trans. Amer. Math. Soc. 112 (1964), 416-431.

5. R. D. Schafer, Nodal noncommutative Jordan algebras and simple Lie algebras of characteristic $p$, Trans. Amer. Math. Soc. 94 (1960), 310-326.

UNIVERSITY OF IOWA 